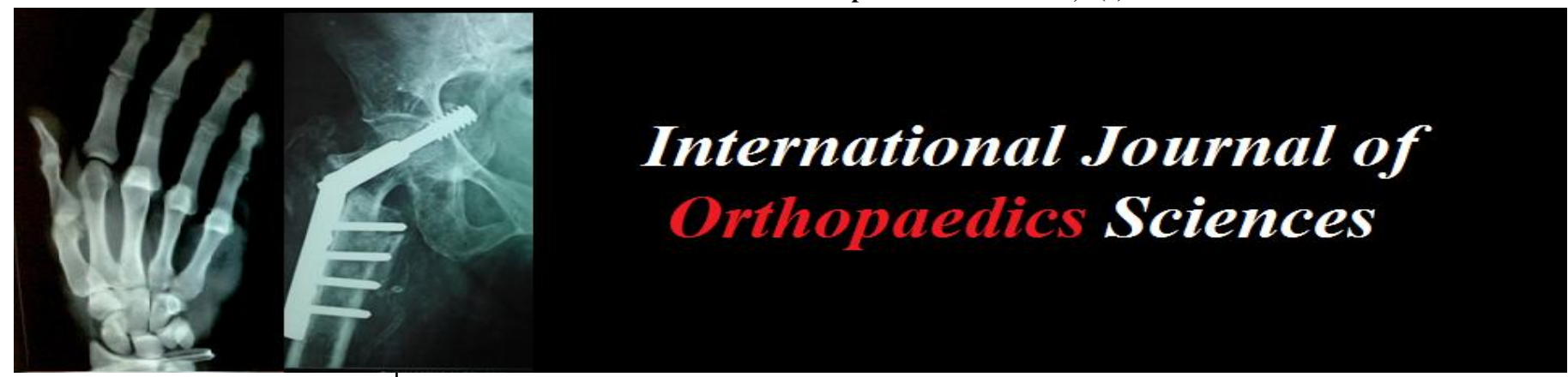

ISSN: $2395-1958$

IJOS 2018; 4(1): 38-40

(C) 2018 IJOS

www.orthopaper.com

Received: 14-11-2017

Accepted: 15-12-2017

\section{Rajni Ranjan}

Associate Professor,

School of Medical Sciences \&

Research, Knowledge Park -3, Greater Noida, Uttar Pradesh,

India

\section{Rakesh Kumar}

Assistant Professor,

School of Medical Sciences \&

Research, Uttar Pradesh, India

Ajeet Singh

Consultant Orthopaedic Surgeon,

R.K. Lifeline, Sirsa, Haryane, India
Correspondence

Rajni Ranjan

Associate Professor,

School of Medical Sciences \&

Research, Knowledge Park -3,

Greater Noida, Uttar Pradesh,

India
Original Research Article

\section{Arthroscopic reconstruction of anterior cruciate Ligament injury with autogenous hamstring graft and functional recovery of the patients}

\section{Rajni Ranjan, Rakesh Kumar and Ajeet Singh}

DOI: https://doi.org/10.22271/ortho.2018.v4.i1a.09

\section{Abstract}

Introduction: Arthroscopic Anterior Cruciate Ligament reconstruction is common surgery in orthopaedics. Hamstring graft is commonly used with excellent results. Aim of our study was to review functional outcome of patient who had undergone ACL reconstruction using Hamstring autograft.

Material and method: A prospective study was done and patient included in the study on the basis of inclusion and exclusion criteria. Arthroscopic ACL reconstruction done with autogenous hamstring graft. At final follow-up functional assessment was done according to Lysholm and Tegner Knee Scoring system.

Result: Total 20 patient included in the study, with age range of 20-56 years. There were 17 male and 3 female. Road traffic accident was most common mode of injury in our study. Lysholme and Tegner Knee Score were excellent in 15 and good in 5.

Conclusion: Arthroscopic ACL reconstruction with autogenous hamstring graft give high success rate in terms of functional recovery of patients.

Keywords: Anterior Cruciate Ligament, Hamstring Graft, The Bone Patellar tendon bone graft, Lysholm and Tegner Knee Scoring system, Osteoarthritis

\section{Introduction}

Anterior Cruciate Ligament (ACL) Injuries are the commonest among ligamentous injuries of the knee. ${ }^{[1]}$ ACL injuries are common among athletes whose sports involves with knee pivoting movement such as football. ${ }^{[2,3]}$. As this condition mainly affect young individual who practice sports, the treatment provided must allow them to return their sports early. ${ }^{33}$. Conservatively treated Patient has unstable knee $s$ with increased anterior laxity and higher risk of severe degenerative arthritis of knee joint. ${ }^{[4]}$ Reconstruction to ACL allows the patient to return to the pre trauma activity level and delay the onset of tibio-femoral degenerative arthritis ${ }^{[2]}$. ACL reconstruction improve stability and function of the knee and reduces the risk of long term cartilage and meniscal injuries. ${ }^{[5]}$. The quadruple hamstring tendon (Semitendinosus-Gracilis) graft has excellent material strength, minimal effect on the knee extensor mechanism and excellent postoperative recovery. ${ }^{2]}$

Aim of our study was to review functional outcome of patient who had undergone ACL reconstruction using Hamstring autograft.

\section{Material and Method}

This prospective study was done in School of Medical Sciences and Research and associated Sharda Hospital, Greater Noida, from January 2013- December 2016. The patients were included in the study on the basis of inclusion and exclusion criteria.

Inclusion Criteria

1. Skeletally Mature Patient

2. Positive Lachman's test

3. Unilateral ACL tear

4. MRI Confirmed ACL tear

5. Patient willing to undergo post-operative rehabilitation protocol

6. Less than 1 year since injury 


\section{Exclusion Criteria}

1. Anterior cruciate Ligament Avulsion injury

2. Previous surgery in same knee

3. Associated MCL or LCL tear

4. Associated injury of any other joint of lower limb

Technique: All patients operated under spinal anesthesia. Under anesthesia through clinical examination of knee was done to confirm ACL tear and to rule out any other concomitant ligaments and collaterals tears. Limb was elevated for 5 minutes before inflation of tourniquet.

Graft harvesting: In all cases we used hamstring grafts. 3 to $4 \mathrm{~cm}$ longitudinal incision was given over the anteromedial aspect of tibia, $3 \mathrm{~cm}$ below the joint line and $2 \mathrm{~cm}$ medial to tibial tubercle. Sartorial fascia identified incised proximally parallel to the tendons. Semitendinosus and gracilis tendons identified by rolling under the finger. After releasing tendons from fascia blunt finger dissection done to free the graft from all fascial bands. Tendon freed from tibial attachments. Tendon ends secured with loop sutures to prevent retraction. Graft harvested with help of closed tendon stripper. Graft is cleared from any muscle and prepared by whipstiching the each end with ethibond no 5 suture. The two strands are looped around a suture to get a quadrupled graft and final length and diameter of graft measured.

Portal placement: anterolateral portal for viewing is made in standard method near to patellar tendon $1 \mathrm{~cm}$ above the joint line in 90 degree of knee flexion. Medial portal made with help of spinal needle without injuring articular cartilage and medial meniscus.

Notch preparation and femoral tunnel placement: Femoral notch (laterally) cleaned with help of shaver the femoral foot print identified. We prepare femoral tunnel before tibial tunnel. For femoral tunnel preparation knee flexed more than 90 degree. The intraosseous tunnel length is measured. It is desirable to keep at least $20 \mathrm{~mm}$ of the graft in the femoral tunnel to allow secure bio integration of graft.

Tibial tunnel: Tibial tunnel is placed in the Centre of tibial foot print. Jig is placed through anteromedial portal and guide pin is drilled into the articular surface under vision. Tibial reamer is used next to create a tunnel of size based on the graft size.

Final preparation of graft and passage: we used the fixed length looped button with graft depending on tunnel length. We put the mark on graft to confirm adequate intraosseous portion. The implant usually suddenly gives way indicating that it has flipped outside the cortex which can be confirmed by pulling back the graft.

Graft tensioning and tibial fixation: The graft is now tensioned by applying gentle traction and taking the knee through15-20 cycles of flexion and extension. We fixed the tibial side of graft in 20 degree of knee flexion by bioabsorbable or metallic screw one size larger than tunnel size. The joint is inspected for any impingement and smooth movement of graft. Through lavage of joint done at end of procedure.

Post-operative protocol: Patient was allowed to bear weight with crutches with long knee extension brace on $2^{\text {nd }}$ post- operative day or on the basis of pain tolerance of patient. Close chain exercise started at 2 week.

The patient was followed up at 6 week, 3 month, 6 month 9 month and 1 year. At final follow-up functional assessment done according to Lysholm and Tegner Knee Scoring system. $[6,7]$

\section{Result}

Out of 20 patients 17 were male and 3 were female with 12 right knee and 8 left knee. The mean age of patient was 31.6 years with age range 20-56 years. Most of the patient $(n=10)$ were in age range of 20-30 years. Most common mode of injury was Road Traffic Accident $(n=10)$ followed by sports injury $(n=6)$. Average time between injury and surgery was 18 weeks. In one patient there was anterior cortex blowout of tibial screw fixation which was revised on next day of surgery, with new tibial tunnel preparation. One patient developed infection in immediate post-operative period which resolved on intravenous antibiotic based on culture sensitivity report. No patient developed any graft donor site related complications. None of patients developed knee stiffness. All patients had full range of movement knee at one year followup. Lysholme and Tegner Knee Score were excellent in 15 and good in 5 .

\section{Discussion}

The ACL has poor potential for spontaneous healing when it is completely torn. Two third of patients develops knee instability following injury to ACL. Conservative treatment may function well in those who are minimally exposed to high risk activities or an advanced process of degenerative arthritis in the knee already had taken place. [3] Surgical reconstruction is the gold standard for the treatment of ACL injury in sportsman and young individual in achieving high rate of success in terms of recovery of normal knee function and return of pre injury level of activity. ${ }^{[8,9]}$ Reconstruction of ACL allow the patient to resume sporting activity and delay the onset of osteoarthritis. ${ }^{[10]}$ Three autografts are commonly available for ACL reconstruction. The Bone Patellar Tendon Bone (BPTB) allows for bone to bone healing within femoral and tibial tunnel and has theoretical advantage of fast healing. Semitendinosus and gracilis tendon has less donor site morbidity compared to BPTB graft. Third option is the quadriceps tendon. ${ }^{[11]}$ There is conventional single bundle reconstruction technique which involves placing single bundle of graft in a tunnel whereas double bundle reconstruction involves two graft bundle in separate tunnel and is considered more anatomical. ${ }^{[12]}$ Age is important key factor influencing results, younger patient show better functional score and a rapid return of activity. ${ }^{[8]}$

Table 1: Age Distribution

\begin{tabular}{|c|c|c|}
\hline Age Group & NO & Percentage \\
\hline $20-30$ & 10 & $50 \%$ \\
\hline $31-40$ & 7 & $35 \%$ \\
\hline $41-50$ & 1 & $5 \%$ \\
\hline $51-60$ & 2 & $10 \%$ \\
\hline
\end{tabular}

Table 2: Mode of Injury

\begin{tabular}{|c|c|c|}
\hline Mode & No & Percentage \\
\hline Road traffic Accident & 10 & $50 \%$ \\
\hline Sports & 6 & $30 \%$ \\
\hline Fall & 4 & $20 \%$ \\
\hline
\end{tabular}


Table 3: Functional outcome (Lysholm \&Tegner Knee Score)

\begin{tabular}{|c|c|c|}
\hline Score & No of Patient & Percentage \\
\hline Excellent & 15 & $75 \%$ \\
\hline Good & 5 & $25 \%$ \\
\hline Fair & 0 & $00 \%$ \\
\hline
\end{tabular}

\section{Conclusion}

Arthroscopic ACL reconstruction with autogenous hamstring graft is an excellent procedure. High success rate has been achieved after this procedure with regard to functional recovery of patients.

\section{Reference}

1. Desai N, Björnsson, Samuelsson K, Karlsson J, Forssblad M. Outcomes after ACL reconstruction with focus on older patients: results from The Swedish National Anterior Cruciate Ligament Register. Knee Surg Sports Traumatol Arthrosc, 2014; 22:379-386.

2. Robindro P, Latchumi NVK, Kanthimathi BD. To study the functional outcome of arthroscopic ACL reconstruction using hamstring graft fixed with endobutton for femur and interference screw and suture post for tibial fixation. IJMDS, www.ijmds.org, 2016, 5(1).

3. De Souza MG, Pampolha, AGM, Junior NO. Functional results from reconstruction of the anterior cruciate ligament using the central third of the patellar ligament and flexor tendons. Rev. bras. ortop. 2015; 50(6):705-71.

4. Mihelic R, Jurdana H, Jotanovic Z, Madjarevic T, Tudor A. Long-term results of anterior cruciate ligament reconstruction: a comparison with non-operative treatment with a follow-up of 17-20 years. International Orthopaedics (SICOT), 2011; 35:1093-1097.

5. Bonasia ED, Dettoni F, Bruzzone M, Bertolo C, Rossi R. Single bundle anatomic anterior cruciate ligament reconstruction: Indications, technique and results. OA Sports Medicine 2013; 01;1(2):13.

6. Tegner Y, Lysholm J. Rating systems in the evaluation of knee ligament injuries. Clin Orthop Relat Res. 1985; (198):43-9.

7. Mitsou A, Vallianatos P, Piskopakis N, Maheras S. Anterior cruciate ligament reconstruction by over-the-top repair combined with popliteus tendon plasty. J Bone Joint Surg Br. 1990, 72(3).

8. Francesco. cruciate ligament reconstruction and rehabilitation: predictors of functional outcome. Joints 2015, 3(4).

9. Almeida A. Evaluation of the results of arthroscopic acl reconstruction with autogenous flexor tendons. Revista Brasileira de Ortopedia, 2010; 45:4.

10. Razi M, Sarzaeem MM, Kazemian GH, Najafi F, Najafi MA. Reconstruction of the anteriorcruciate ligament: a comparison between bone-patellar tendon-bone grafts and four-strand hamstring grafts.Med J Islam Repub Iran, 2014, 28.

11. Macaulay AA, Perfetti DC, Levine WN. Anterior Cruciate Ligament Graft Choices. Sports health. 2012; 4(1):63-68.

12. Morey VM, Nag HL, Chowdhury B, Sankineani SR, Naranje SM. A prospective comparative study of clinical and functional outcomes between anatomic double bundle and single bundle hamstring grafts for arthroscopic anterior cruciate ligament reconstruction. International Journal of Surgery, 2015; 21:162-167. 